\title{
Digital Service Model of Red Educational Resources from the Perspective of Excellent Traditional Culture
}

\author{
Dan Liu \\ School of Law, Shangqiu Normal University, Shangqiu, Henan 475000, China \\ Correspondence should be addressed to Dan Liu; liudan@sqnu.edu.cn
}

Received 1 December 2021; Revised 30 December 2021; Accepted 26 January 2022; Published 24 February 2022

Academic Editor: Wei Zhang

Copyright (C) 2022 Dan Liu. This is an open access article distributed under the Creative Commons Attribution License, which permits unrestricted use, distribution, and reproduction in any medium, provided the original work is properly cited.

\begin{abstract}
In view of the poor service effect caused by the incomplete index base of the traditional red education resources digital service model, this paper puts forward the red education resources digital service model from the perspective of excellent traditional culture. Determine the influencing factors of red education resources in the integration process, integrate the remote scheduling principle of teaching information into the feature vector extraction of red education resources, extract the number of main factors of the feature vector, decompose the feature vector of red education resources, take the wavelet entropy of red education resources as the fusion weight, and integrate the obtained coefficients through wavelet transformation. Get the integration results of red education resources. Build the architecture of digital service model, grab all web pages on the Internet with indexer, and create forward index and reverse index. Use the controller to search web pages and control all web pages to be searched. Restrict query conditions through user interface. Establish a red education resource data index database, query and process documents, and build a digital service model. The experimental results show that the designed service model has good goodness of fit and high performance.
\end{abstract}

\section{Introduction}

In the process of building the digital service model, attention to the digital service of the revolutionary and traditional red education resources in education is put on the agenda $[1,2]$. In order to meet the digital needs of civic education, reflect the personalized services of characteristic education resources from the perspective of excellent traditional culture in civic education, and give play to the application of information technology in the "moral education first" strategy of main education; the digital construction and application of red education resources have gradually attracted the attention of schools and society [3]. Red education resources refer to the great revolutionary spirit and its carrier formed by the Communist Party of China leading the Chinese people to struggle for many years during the revolutionary period, and are precious resources for the education of loving the party, patriotism, and socialism in the new period $[4,5]$. However, due to the influence and limitation of geographical environment and other technical factors for a long time, red education resources cannot be comprehensively applied and shared [6]. Whether the Internet can be used to share the red education resources publicly to the society, so that more people can use modern media technology to share the red education resources, is a new subject facing ideological education and educational technology workers [7]. Therefore, it is necessary to systematically analyze and study the digital construction and effective application of red educational resources, in order to promote the maximum range and maximum degree of open sharing of high-quality characteristic educational resources. Reference [8] proposed open educational resources in a global context. Reference [9] puts forward the path of integrating red cultural resources into socialist core values education of college students under the "micro" background. Based on the above research, the digital service model of red education resources from the perspective of excellent traditional culture is proposed to promote the maximum range and maximum openness and sharing of high-quality characteristic red education resources. 


\section{Red Education Resource Integration Technology Design}

2.1. Determine the Factors Affecting the Integration of Red Education Resources. During the integration of red education resources, from the perspective of excellent traditional culture, based on the remote scheduling theory of teaching information, different quantitative values existing in the integration of red education resources are obtained, and the factors affecting the integration of red education resources are extracted. These factors mainly include the effect of red education, students' subjective will to learn, teaching effect, subjective action, and teaching methods.

Determine the factors affecting the integration of red education resources, and the specific process is as follows:

Assuming that $s$ represents the factors that various conflicting events affect the integration of red education resources, $x$ represents the abnormal measurement value of red education resources in the integration process, $n$ represents the time limit of red education resources in the integration process, and $j$ represents the quantitative value of the integration performance of red education resources. Combined with the remote scheduling theory of teaching information [10], the red education resources are obtained in the integration process. Due to the gap of educational resources, the quantitative value of red educational resources integration is calculated as follows:

$$
O_{h}=\sum_{j=1}^{n} \frac{c_{k h} \times v_{s}}{x} .
$$

In the formula, $c_{k h}$ represents the conventional performance of red education resources in the integration process, and $v_{s}$ represents the adaptability of red education resources in the integration process. Then the integration probability of red education resources can be calculated by using formula (2), which is

$$
\vartheta\left(V_{y}\right)=\frac{\mu_{b} \times \kappa_{r}}{O_{h}} \times c_{k h}
$$

In the formula, $\kappa_{r}$ represents the integration status of red education resources, and $\mu_{b}$ represents the change characteristics of red education resources in the process of integration. Let $h_{r}$ indicate the characteristics of red education resources and determine the influencing factors of red education resources in the process of integration. The formula is

$$
K_{v}=\frac{h_{r} \times z_{o}}{\tau_{m}} \times \frac{F^{D}}{\iota(i) \times a_{d}} .
$$

Among them, $\iota(i)$ represents the attributes of red education resources, $i$ represents the factor attribute vector affecting the integration of red education resources, $F^{D}$ represents the selected characteristics, $z_{o}$ represents the time required for the integration of red education resources, $\tau_{m}$ represents the failure probability of red education resources in the integration, and $a_{d}$ represents the integration cycle of red education resources.
The result of determining the factors affecting the integration of red education resources is to obtain the quantitative value of the integration of red education resources based on the remote scheduling theory of teaching information and determine the influencing factors formed in the integration process of red education resources by calculating the integration probability of red education resources.

\subsection{Extracting Feature Vectors of Red Education Resources.} In the process of integrating red education resources, the remote scheduling principle of teaching information is integrated into the feature vector extraction of red education resources $[11,12]$, the feature variance contribution rate of red education resources is calculated, the observable random vector of the features of red education resources is given, and the feature vector of red education resources is decomposed by extracting the number of main factors of the feature vector of red education resources [13]. The specific process is as follows:

Assuming that $n$ represents the number of characteristic variables of the original red education resources, $X$ represents the $n$ characteristic variables in the original red education resources samples, which need to meet the conditions of $X=x_{1}, x_{2} \ldots x_{n}$, the red education resources are processed by orthogonal transformation, and $u^{\prime}$ characteristic variables $\left(y_{1}, y_{2}, y_{3}\right)$ and $R$ represent the correlation coefficient matrix of the red education resources samples. Using the teaching information remote scheduling theory, establish the characteristic equation of red education resources, expressed as

$$
\lambda^{n}(i)=\frac{\{R \times X\}^{n}}{\left\{\left(y_{1}, y_{2}, y_{3}\right)\right\}} \times \frac{\left(x_{1}, x_{2} \ldots x_{n}\right)}{u^{\prime}}
$$

Assuming $\lambda_{i}$ represents the number of nonnegative eigenvalues of the correlation coefficient matrix of the red education resource samples, and $\lambda_{i}$ is sorted under the condition of $\lambda_{1} \geq \lambda_{2} \geq \lambda_{n} \geq 0$, the first $m$ red education resource features can be extracted, namely,

$$
\Phi(p)=\frac{m \times\left(\lambda_{i}\right)}{\xi(e)} \times \eta(r) .
$$

In the formula, $\xi(e)$ represents the noise interference of the characteristics of red educational resources, and $\eta(r)$ represents the uncertainty of the characteristic vector of red educational resources. Let $\alpha$ represent the variance of the characteristics of the first $m$ red education resources, and the calculation formula is

$$
\beta(p)=\frac{m \times \alpha}{\mu(R)} \times \nu(e)(\sigma \times \kappa) .
$$

In the formula, $\mu(R)$ represents the weight of characteristic samples of red education resources, $\nu(e)$ represents the information entropy of different characteristics of red education resources, $\sigma$ represents the optimal threshold of characteristic variables of red education resources, and $\kappa$ represents the observation variables of student characteristics. 
$X=x_{1}, x_{2} \ldots x_{n}$ is defined as the random vector of the characteristics of red education resources, $a_{i j}$ represents the factor load of the characteristic vector of red education resources, and the observable random vector of the characteristics of red education resources is calculated, that is,

$$
\partial(X)=\frac{X \times F}{\left(a_{i j}\right)_{n \mp m}} \times c_{i} \times \varepsilon_{i} \times X_{i} .
$$

In the formula, $F$ represents the unobservable vector of red education resources, $c_{i}$ represents the factor load of special red education resources, and $\varepsilon_{i}$ represents the unique factor affecting factor load $c_{i}$. Measure the uncertainty degree of red education resource information source $X^{*}$, and the calculation formula is

$$
H\left(X^{*}\right)=\frac{p_{i} \log p_{i}}{H\left(p_{1}, p_{2}, \cdots, p_{n}\right)} I\left(a_{i}\right)
$$

In the formula, $I\left(a_{i}\right)$ represents the space of red education resource information source $X^{*}, p_{i}$ represents the probability of occurrence of red education resource integration strategy, and $H\left(p_{1}, p_{2}, \ldots, p_{n}\right)$ represents the probability of occurrence of discrete random variables. Let $I\left(\lambda_{i}\right)$ represent the information function of red education resource integration, and the feature vector of red education resource integration behavior class can be defined as

$$
\xi(w)=\frac{I\left(\lambda_{i}\right) \times H\left(X^{*}\right)}{\zeta(k)} \times 9(R) .
$$

In the formula, $\zeta(k)$ represents the information amount of red education resources and $\vartheta(R)$ represents the cumulative information contribution rate of red education resources integration.

The result of extracting the feature vector of red education resources is to integrate the remote scheduling principle of teaching information into the feature vector of red education resources, calculate the feature variance contribution rate of red education resources, extract the number of main factors of the feature vector of red education resources, decompose the feature vector of red education resources, and lay a foundation for the integration of red education resources.

\subsection{Integrating Red Education Resources. According to the} extraction results of red education resource feature vector, a complete red education resource data set is established. Through the data fusion algorithm [14, 15], all the red education resource features in the red education resource data set are reconstructed [16], and the reconstructed red education resource data set is decomposed. According to the decomposition results, the wavelet variance of red education resources is calculated [17]; take it as the weight fusion wavelet coefficient to obtain the integration result of red education resources. The specific process is as follows:

Step 1: Assuming $q$ resource managers manage multiple red education resources $X$ at the same time, the red education resource reserve value in each resource manager can be expressed as

$$
Z_{i}=X+V_{i}, i=1,2, \ldots, m .
$$

In the formula, $V_{i}$ represents the red education resource parameter in the resource manager. Suppose $\delta_{i}$ represents the weight in each resource manager and $\sigma_{i}$ represents the variance of red education resource parameters. Finally, the integration value of red education resources is $Z=\sum_{i=1}^{q} \delta_{i} Z_{i}$, and the total mean square error of red education resource integration is

$$
\begin{aligned}
\sigma^{2} & =E\left[(X-Z)^{2}\right] \\
& =\sum_{i=1}^{q} \delta_{i}^{2} \sigma_{i}^{2} .
\end{aligned}
$$

Step 2: According to the extreme value theory of multivariate function, it can be calculated that the minimum value of formula (11) is $\left(1 / \sum_{i=1}^{q}\left(1 / \sigma_{i}^{2}\right)\right)$, and the weighting factor corresponding to the minimum value is

$$
\delta_{i}=\frac{\left(1 / \sigma_{i}^{2}\right)}{\sum_{i=1}^{q}\left(1 / \sigma_{i}^{2}\right)} .
$$

Step 3: The weighting factor obtained by formula (12) is used to reconstruct the characteristics of red education resources. The formula is

$$
Z_{J}=\sum_{k} h_{n}(J, k) \varphi_{J, k}+\sum_{J} \sum_{k} g_{n}(J, k) \psi_{J, k}
$$

In the formula, $Z_{J}$ represents the reconstructed red education resources, $\varphi_{J, k}$ represents the scale function of the red education resources in the $J$ layer, $h_{n}(J, k)$ represents the $k$ approximation coefficient of the red education resources, $\psi_{J, k}$ represents the wavelet resource function of the red education resources in the $J$ layer, and $g_{n}(J, k)$ represents the $k$ detail resource function of the red education resources. Assuming that $W_{h, i}$ represents the weighting factor of the approximation parameter of red education resources and $W_{g, i}$ represents the weighting factor of the detail parameter of red education resources, the following is obtained:

$$
\begin{aligned}
\sigma^{2} & =\sigma_{h}^{2}+\sigma_{g}^{2} \\
& =E\left[Z_{V}^{2}-\sum_{i=1}^{q} W_{h, i}^{2} \frac{1}{2} \eta_{i} \varphi_{J, k}\right] .
\end{aligned}
$$

Step 4: According to formula (14), the mean square deviation of the weighted result of the integration of red education resources is less than or equal to the mean square deviation of the optimal weighting of red education resources in time domain. Through the remote scheduling of online teaching resources through teaching information $[18,19]$, the mean square deviation of red education resources becomes smaller and smaller with the decomposition of scale. The final conclusion provides a theoretical basis for the integration of red education resources [20-22]. 
Step 5: The wavelet entropy of red education resources is obtained by using the distribution of energy series of red education resources [23-25]. The specific process is as follows:

$$
H_{\mathrm{we}}=H\left(p_{1}(E), p_{2}(E), \ldots, p_{J}(E)\right) .
$$

In the formula, $p_{J}(E)$ represents the normalized energy sequence of red education resources. To sum up, the wavelet entropy of red education resources is taken as the fusion weight of red education resources, and the coefficient obtained is integrated. After wavelet transformation, the integration result of red education resources is obtained, and the red education and teaching resources are integrated to build a harmonious campus, so as to create a good environment for the healthy growth of college students, all-round, multilevel shape and cultivate college students' correct outlook on life, world outlook, and values with high taste, vigorously carry forward the positive, healthy, and upward red spirit, highlight the red melody representing the development direction of the times and reflecting the requirements of social progress, enhance the effectiveness of red education resources teaching, and realize the integration of red education resources.

\section{Red Education Resources Digital Service Model Architecture}

3.1. Digital Service Model Architecture. The basis for establishing the architecture of digital service model is that the system is the core of the construction of teaching environment. It is based on digitization, takes disciplines and majors as the main line, takes courses as the center, and integrates teaching document information such as teaching plan, curriculum, and syllabus, as well as various types of teaching resources such as teachers' lectures, courseware, reference materials, and multimedia resources. The integrated resources are organically run through the teaching process to provide teachers and students with all-round and practical teaching information and teaching reference resources services. In the view of students, a large amount of redundant information brought by search engines wastes a lot of students' time. At the same time, due to the huge amount of information, it also adds a lot of inconvenience to software developers to find relevant information [26, 27]. In order to make better use of the existing red education resources and meet people's needs for information retrieval, there is an urgent need for a digital basic education search engine. The architecture of digital service model is shown in Figure 1.

According to Figure 1, the structure of digital service model is mainly composed of indexer, controller, user interface, and other key parts. The architecture of digital service model takes digital technology as the core and digital industrialization as the main line. According to the requirements of colleges and universities for large databases, a systematic, more applicable data platform that can provide accurate data information is gradually established to promote front-end application and promote the all-round development of students. The contents of each component are described in detail below.

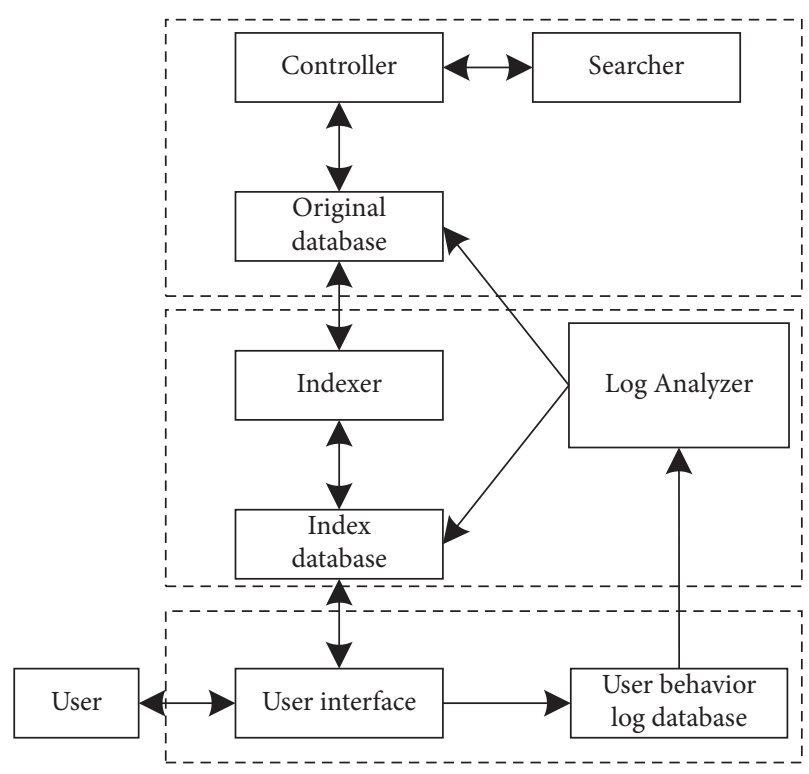

FIgURE 1: Architecture of digital service model.

3.1.1. Indexer. It is a web spider that searches for web page link addresses in a web page, reads the content of the web page from a page of the website (usually the home page), looks for other link addresses in the web page, and then finds that the next page enters through these link addresses. This cycle will continue until all pages of the site are crawled [28]. Taking the whole Internet as a site, web spiders can use this principle to grab all web pages on the Internet. The main function of the indexer is to obtain the information of teaching materials, extract the index items, use them to represent the digital documents of red educational resources, and generate the index table. For search engines, selecting index words and keywords is a more core problem [29]. When common words are selected as index words in search engines, although the storage required for retrieval is small, the frequency of each index word is high. Because there are many unregistered words, it is obviously not suitable to introduce them into the index vocabulary. Therefore, use statistical methods to filter unregistered words.

Statistical vocabulary acquisition is another method to identify unregistered words. In large corpora, words with certain expression ability often do not appear in isolation but have certain statistical rules. The statistical method is used to automatically obtain vocabulary by using the cooccurrence information between words. Formally, a word is a stable combination of words. Therefore, the more the adjacent words appear at the same time in the context, the more likely it is to form a word [30]. Therefore, the frequency or possibility of word combination can better reflect the reliability of word formation $[31,32]$. The combination frequency of adjacent cooccurrence symbols and their mutual occurrence information are calculated in the corpus. The mutual information between two Chinese characters is determined, and their adjacent cooccurrence probability is calculated.

Reciprocal information is the embodiment of the compact combination of Chinese characters. If the tightness 
exceeds a certain threshold, it can be considered that this phrase constitutes a word. After analyzing web pages and word segmentation, we need to use inverse indexing technology to index word segmentation. Establishing reverse indexes includes establishing forward and reverse indexes, as shown in Figure 2.

As shown in Figure 2, after analyzing the web page, you get a forward index table with the page number as the primary key. To speed up the recombination process, the whole process must be done in memory. In the case of large data volumes, there is enough memory to ensure that the creation process is completed in one go. After increasing the amount of data, you can group data and then merge data. The index module's strategy is to divide the index into $\mathrm{K}$ groups, depending on the memory size of the computer system, so that each set of operations requires less memory than the maximum memory size that the system can provide. The inverted index of group $\mathrm{K}$ is generated by using the inverted index generation algorithm. The inverted index of group $\mathrm{K}$ is merged, and the data corresponding to the same index is merged together. Finally, the inverted file index with the index as the primary key is obtained, that is, the inverted index.

3.1.2. Controller. The controller focuses on solving the overall efficiency and quality problems. The so-called efficiency is to collect predetermined web pages with as few resources, computer equipment, network bandwidth, and time as possible $[33,34]$. Even when using computers to collect web pages, we should also pay attention to parallel development and utilization. While arranging to use multiple computers to form a cluster, share the exported network bandwidth. With the increase of the number of devices, the network bandwidth domain quickly becomes the bottleneck of the environment. In addition, it may be too late to provide the required web pages on the server side, so do not let the crawling process started by the collector focus on a few sites. If you pay too much attention to the collection activities of some websites, or grab too many pages from a website in a short time, it may lead to quality problems. Web crawler can not grab web pages too frequently, which will affect the normal access of website users. Because the number of web pages collected in a certain period of time is limited, search as many important web pages as possible and do not miss important web pages [35]. Identifying the importance of web pages is also the key to structure mining. Using the home page as much as possible and then conducting the first round of search on the home page can improve the overall search efficiency.

3.1.3. User Interface. The function of user interface is to input user query, display query results, and provide user relevance feedback mechanism. Its main goal is to facilitate users to use search engine and obtain efficient and timely information through various ways, so as to improve efficiency. The design and implementation of users adopt the theory and method of human-computer interaction, which

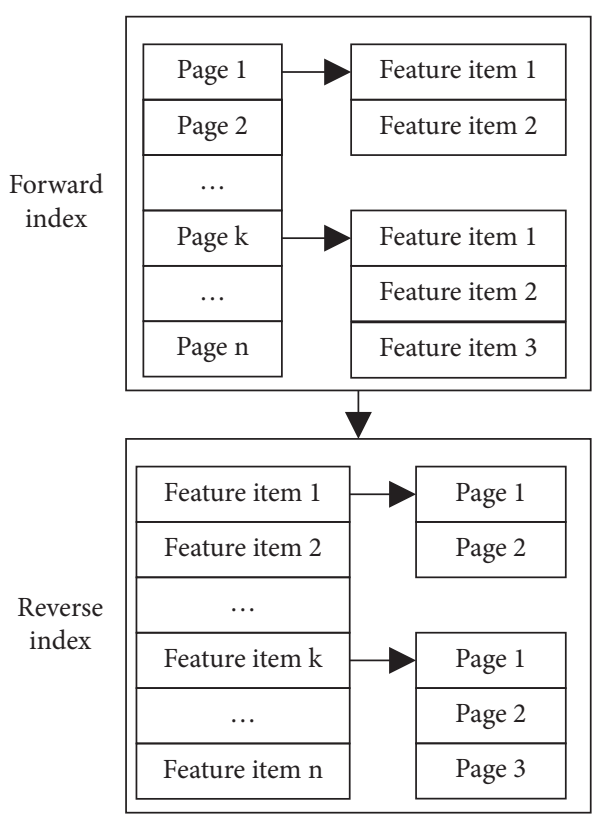

FIgURE 2: Establishing reverse index from forward index.

is fully in line with human thinking habits. Through the user interface of intelligent search engine, users can submit query requests in natural language. The system will decompose the word segmentation system according to the needs of users to obtain the words to be queried [36]. The user input interface can be divided into simple interface and complex interface. A simple interface provides a text box for users to input query strings, while a complex interface allows users to limit query conditions, such as logical operation, similarity, domain name range, location, information display time, length, etc.

\subsection{Service Process Design}

3.2.1. Construction of Red Education Resource Data Index Database. The process of establishing the data index database of red education resources in the query system is as follows (Figure 3).

Step 1: First, the user gives the query conditions.

Step 2: The index system searches the document subset related to the query requirements according to the query requirements of excellent traditional culture.

Step 3: According to the subset of red education resource documents, check whether the obtained digital documents of red education resources are related to the query criteria.

Step 4: If the document is not related to the query criteria, return to Step 2. If the document is related to the query criteria, the relevance is sorted.

Step 5: Finally return the red education resource document and the document sorted by query criteria to the user. Figure 3 shows a data index database of red education resources. 


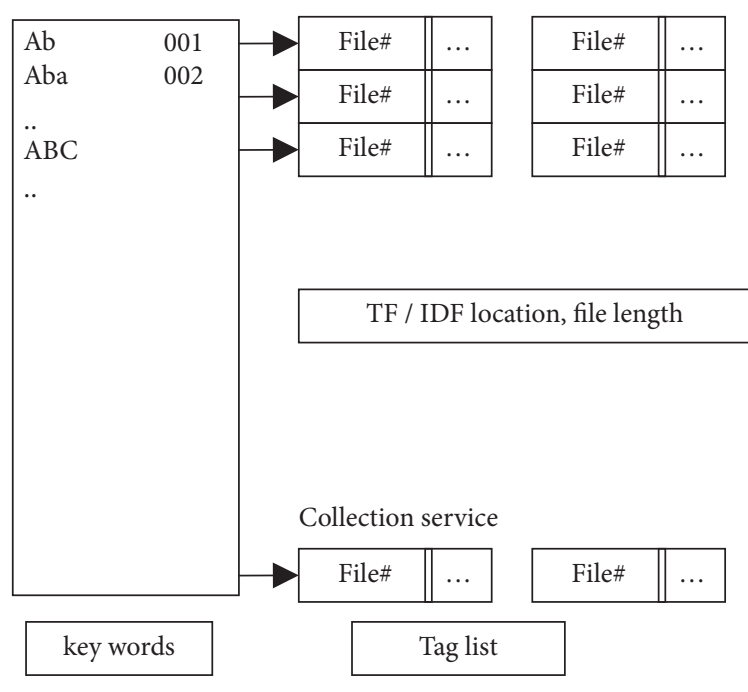

Figure 3: Red education resource data index database.

With the support of the red educational resource data index library shown in Figure 3, determine the factors affecting the index and query the number of keywords, connection operation fields, and values in the range. Probability distribution of values in the value field, field update frequency, index maintenance cost, and database insert and delete operation frequency. Based on these factors, it is determined to build index on the field, so as to complete the construction of red education resource database index.

\subsubsection{Query and Processing of Digital Documents of Red} Education Resources. Before creating the index, the statistical component of the digital document of red education resources needs to summarize and record the statistical data corresponding to the text features and then use these data to calculate the score of the digital document of red education resources. According to the sorting algorithm and retrieval model, determine the data users want to obtain. The specific process is shown in Figure 4.

According to Figure 4, the specific steps of querying and analyzing the digital documents of red education resources are as follows.

(1) Analyze and query digital documents of red education resources. Firstly, the analysis and processing of the query correspond to the processing steps of the digital document of red education resources; that is, the words in the query are converted into the same form as the words generated when processing the text of the digital document of red education resources. Otherwise, an error will occur when sorting. Text analysis mainly includes lexical analysis, that is, to identify the morpheme information, vocabulary information, and phrase information contained in the text content. The result of file analysis is the representation of the corresponding structure and related content of the file.

(2) Remove stop words. Stop words are high-frequency words or text information, such as prepositions used in digital documents of red education resources. These two function

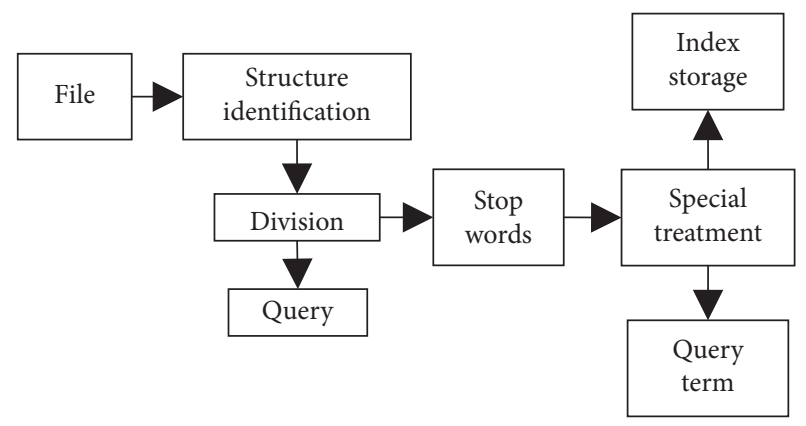

FIGURE 4: Query and analysis of digital documents of red education resources.

words are helpful to sentence structure but also help to describe the topic in the article. Removing these two words can not only reduce the size of the index, but also reduce the occupation of corresponding memory space and improve the speed and effect of the index.

(3) Extract stem. In the process of retrieval, stem extraction can match information retrieval with related semantics. If a word is deformed or derived from multiple forms, it can be simplified to the same stem.

3.2.3. Construction of Digital Service Model. To save space, use the smallest integer data possible. In larger tables, the number of bytes grows very fast. On the other hand, once a field is created, it is very difficult to modify it. Therefore, for security reasons, you should predict the maximum value of the field that may need to be stored and then select the appropriate data type. To better control the data stored in the field, numerical data can be used to represent the integer part and decimal part of the number, and a service model is established based on this.

Let the sample set be $X=\left\{x_{1}, x_{2}, x_{3}, \ldots \ldots, x_{n}\right\}$ and the estimated parameter be $\alpha$, so as to determine the implied variable. The likelihood function formula is

$$
L(\alpha)=L\left(x_{1}, x_{2}, x_{3}, \ldots, \ldots, x_{n} ; \alpha\right) .
$$

In formula (16), $L(\alpha)$ represents the likelihood function of parameter $\alpha$ relative to sample set $\mathrm{X}$, and the mathematical expression is

$$
\alpha=\arg \max l(\alpha)
$$

In formula (17), the logarithm of $l(\alpha)$ function is transformed into logarithm summation calculation method to obtain the expression of log likelihood function:

$$
H(\alpha)=\sum_{i=1}^{n} \ln p\left(x_{i} ; \alpha\right)
$$

The digital service model is obtained according to the expression of log likelihood function:

$$
\mathrm{P}=\frac{\left|R_{H(\alpha)} \cap T\right|}{\left|R_{H(\alpha)}\right|} \times 100 \%
$$

In formula (19): $R_{a}$ represents the service result of required red education resources; $T$ represents the required 
sample set. The higher the value, the more accurate the service results will be. The database index service will be completed, so as to complete the construction of the digital service model of red education resources from the perspective of excellent traditional culture.

\section{Experimental Analysis}

The experiment of the digital service model of red education resources from the perspective of excellent traditional culture is verified on the basis of the file archiving and file retrieval performance of the prototype system, which involves each specific functional module. The model is developed in Java language and the operating system is Windows 10. According to the actual application, the resource digital service model is tested in a university laboratory, and four hosts are selected to set the network environment. The specific settings are shown in Table 1.

The experimental data mainly use text and video files, and about 80000 different kinds of files are collected. Different file compression methods are adopted for different file types, which is one of the most effective methods to improve compression efficiency. It can be seen that the decompression time is significantly less than the file compression time. It is precisely because multiple sliding windows are involved in file compression in the digital service model of red education resources, and the internal resources of the window need to be matched with the dictionary. String matching is the most time-consuming, and string matching is not required when decompressing the file.

According to the above design ideas, the model is applied to the real digital service environment of red education resources. The architecture of the model is divided into two phases, and the content of phase I is selected as the research object. The specific work arrangements for the functional requirements investigation, $R \& D$, testing, joint commissioning, functional verification and training, and online trial operation stages are as follows:

(1) Demand research stage: complete demand research, study business model, conduct demand research and analysis, and prepare demand description scheme according to the needs of customers.

(2) Function $R$ and $D$ stage: Design front-end UE interface prototype and data dictionary, system integration interface design and $R \& D$, and function $R$ and $D$.

(3) Test and joint commissioning stage: test environment deployment, interface joint commissioning test, and business function joint commissioning test.

(4) Function verification and training stage: training preparation, user operation training, and gray environment for system function verification.

(5) Online trial operation stage: online trial operation scheme preparation, online trial operation application, production environment deployment, and online trial operation.
In combination with the specific contents of the phase I function implementation arrangement of the above red education resources digital service model, from the perspective of excellent traditional culture and according to the construction of grid support tools in the urban area, we choose to popularize and try out the digital service model of red education resources and analyze its trial effect. After completing the construction of the model, the time consumed to complete various businesses and the response time for providing digital services will be recorded, as shown in Figure 5.

From the implementation results obtained in Figure 5, it can be seen that, before and after the application of the model constructed in this paper, both the time consumed to complete various businesses and the service response time are significantly less than before the application of the model. At the same time, before the application of the model, the increase of business volume will affect the service response time and business consumption time to a certain extent. However, after the application of the model, with the increase of business volume, neither of the two parameters shows an obvious trend of increasing, indicating that the problem that the service response time and business consumption time before the application of the model are affected by business volume is solved. At the same time, the above implementation results further prove that, in the perspective of excellent traditional culture, we can further strengthen the digital service quality of red education resources and improve service experience and satisfaction.

In order to further prove the rationality of the digital service model of red education resources from the perspective of excellent traditional culture, the goodness of fit of the technology is judged by relevant test residuals. The calculation formula of goodness of fit is

$$
R^{\text {new }}=1-\frac{Q}{2 \sum \hat{y}_{2}} \times 100 \% .
$$

In the formula, new represents the determination coefficient, $\hat{y}_{2}$ represents the fitting degree, and $Q$ represents the sum of squares of residuals. By comparing the model of [8] and the model of [9], the goodness of fit test results of red education resource integration analysis under three different models are shown in Figure 6.

It can be seen from the results in Figure 6 that the goodness of fit of the red education resources digital service model for red education resources integration from the perspective of excellent traditional culture is better than the other two methods. The reason is that the model gives the correlation between the factors affecting the integration of red education resources and the integration of red education resources by obtaining the quantitative value of the integration characteristics of red education resources, making the goodness of fit of red education resource integration better.

To sum up, after the application of the digital service model of red education resources designed from the perspective of excellent traditional culture, with the increase in business volume, neither of the two parameters shows an obvious increase trend, indicating that the problems existing 
TABLE 1: Experimental environment settings.

\begin{tabular}{lcccc}
\hline Test tool & Memory size/G & Hard disk size/G & Control system & Set up quantity \\
\hline The server & 4 & 500 & Linux & 1 \\
Host A & 2 & 250 & Win10 & 1 \\
Host B & 4 & 300 & Win8 & 1 \\
Host C & 4 & 300 & Win8 & 1 \\
\hline
\end{tabular}

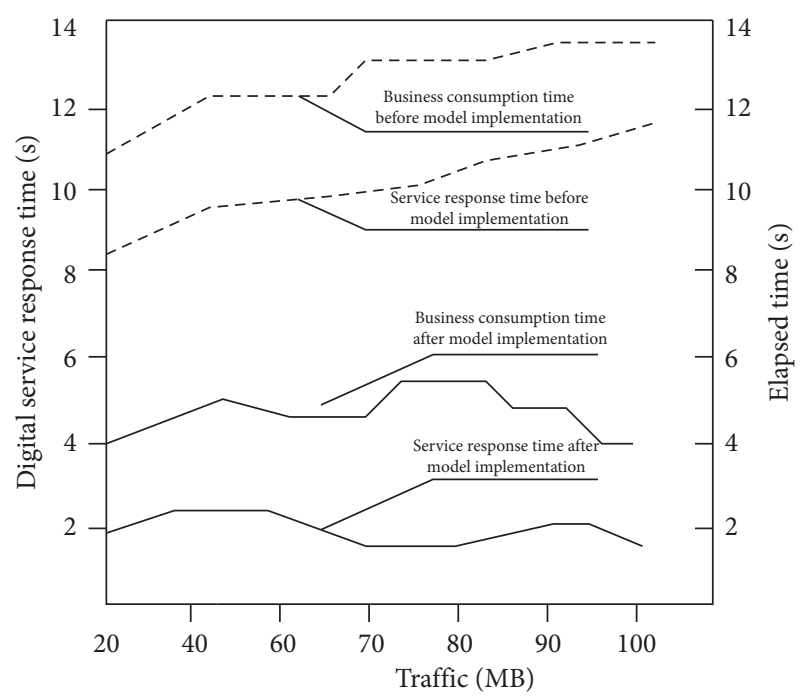

Figure 5: Implementation effect of digital service model of red education resources.

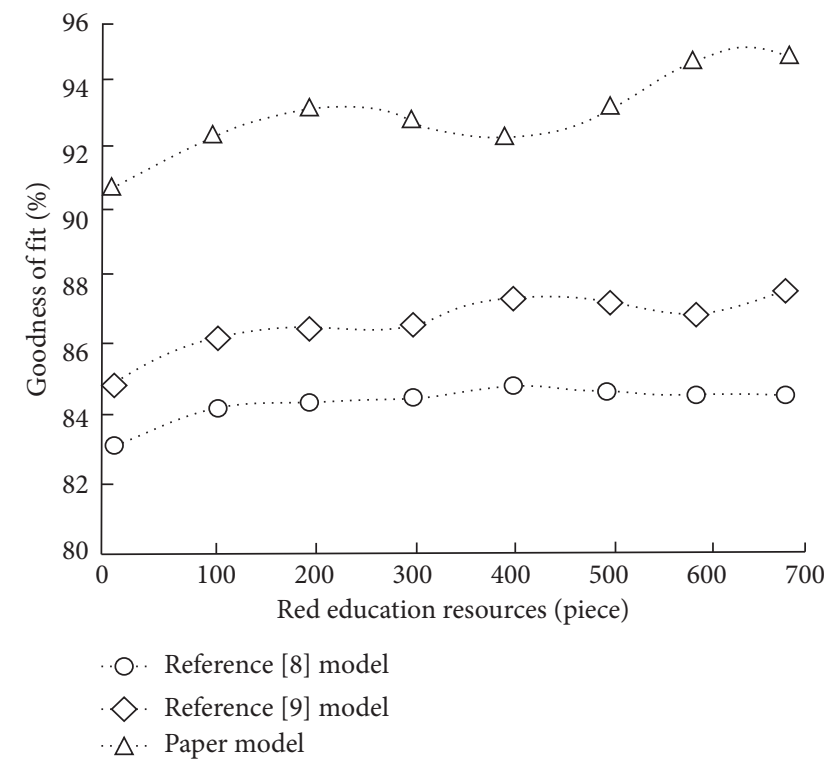

FIGURE 6: Goodness of fit test results of red education resource integration analysis.

before the use of the model are solved and the quality of digital service of red education resources is further strengthened. To improve service experience and satisfaction, the goodness of fit of red education resource integration is better.

\section{Conclusion}

Convenience also provides opportunities and challenges for the digitization and sharing of red education resources. The digital service model of red education resources designed from the perspective of excellent traditional culture consumes significantly less time to complete various businesses and provides service response time. Moreover, the goodness of fit effect of the integration of red education resources is better after the application of the digital service model. There are some deficiencies in the research on the mining and value evaluation of red educational resources, which need to be further improved.

Due to the lack of research level and the limitations of objective conditions, it is necessary to increase research efforts in the future, strengthen communication with relevant local departments, conduct in-depth research on red education resources, and establish a complete library of red education and culture resources. On the other hand, the sample size of index screening and index scoring can be expanded to obtain more objective data as far as possible to ensure scientific research results.

\section{Data Availability}

The raw data supporting the conclusions of this article will be made available by the authors, without undue reservation.

\section{Conflicts of Interest}

The author declares that there are no conflicts of interest regarding this work.

\section{References}

[1] G. Yu and S. O. Marxism, "The revolutionary tradition and contemporary heritage of Chinese youth since the may 4th movement," Contemporary Youth Research, no. 1, pp. 40-44, 2019.

[2] E. Kolchinsky and G. S. Levit, "The reception of Haeckel in pre-revolutionary Russia and his impact on evolutionary theory," Theory in Biosciences, vol. 138, no. 1, pp. 73-88, 2019.

[3] W. Wei and X. University, "Research on outdoor safety education innovation based on jinggangshan red education base," The Theory and Practice of Innovation and Entrepreneurship, vol. 12, no. 6, pp. 15-21, 2019.

[4] M. E. K. Faiz, "The patriotic attitudes of the prospective teachers," International Journal of Psychology and Educational Studies, vol. 7, no. 1, pp. 124-134, 2020.

[5] H. Sockett, "Review of curren and dorn, patriotic education in a global age," Studies in Philosophy and Education, vol. 38, no. 6, pp. 679-682, 2019. 
[6] Y. R. Chassiakos and M. Stager, "Current trends in digital media: how and why teens use technology," Technology and Adolescent Health, no. 1, pp. 25-56, 2020.

[7] S. Xiao, Q. Kong, M. Song, H. Jinde, and C. Jiuling, “Teaching reform of CNC technology course under the ideological and political education," International Journal of Social Science and Education Research, vol. 2, no. 10, pp. 36-41, 2019.

[8] R. Lima-Lopes and T. M. D. Biazi, "Open Education Resources in the global scenario: highlights and challenges," Ilha Do Desterro: A Journal of English Language, Literatures in English and Cultural Studies, vol. 74, no. 3, pp. 12-19, 2021.

[9] S. N. Fang, "Path of integrating red cultural resources into socialist core values education for college students under "micro" background\%," Journal of Zhejiang SCI-TECH University, vol. 42, no. 2, pp. 195-200, 2019.

[10] N. Fujita, "Transforming online teaching and learning: towards learning design informed by information science and learning sciences," Information and Learning Sciences, vol. 22, no. 7, pp. 2398-5348, 2020.

[11] C. R. Kinkar and Y. K. Jain, "WITHDRAWN: feature vector extraction model for noisy reverberant speech signal," Informatics in Medicine Unlocked, vol. 2, no. 7, Article ID 100388, 2020.

[12] A. A. Sekin and M. Sekin, "Detection of fabric defects with intertwined frame vector feature extraction," AEJ - Alexandria Engineering Journal, vol. 25, no. 8, pp. 1-12, 2021.

[13] S. Wang, C. Gao, Q. Zhang et al., "Research and experiment of radar signal support vector clustering sorting based on feature extraction and feature selection," IEEE Access, vol. 8, no. 5, Article ID 93322, 2020.

[14] J. Anees, H.-C. Zhang, S. Baig, B. Guene Lougou, and T. G. Robert Bona, "Hesitant fuzzy entropy-based opportunistic clustering and data fusion algorithm for heterogeneous wireless sensor networks," Sensors, vol. 20, no. 3, pp. 913-941, 2020.

[15] H. Wang, L. Song, J. Liu, and T. Xiang, “An efficient intelligent data fusion algorithm for wireless sensor network," Procedia Computer Science, vol. 183, no. 3, pp. 418-424, 2021.

[16] A. Tsabary and D. Abookasis, "Optimized reconstruction processing of targets hidden in turbid environment from multi-perspective images assisted with sorting algorithmbased quality metrics," Optik, vol. 243, no. 10, Article ID 167349, 2021

[17] D. Luguern, R. Macwan, Y. Benezeth et al., "Wavelet Variance Maximization: a contactless respiration rate estimation method based on remote photoplethysmography," Biomedical Signal Processing and Control, vol. 63, no. 2, Article ID 102263, 2021.

[18] I. I. Suliman, "Estimates of patient radiation doses in digital radiography using DICOM information at a large teaching hospital in Oman," Journal of Digital Imaging, vol. 33, no. 4, pp. 64-70, 2019.

[19] Z. Qian, A. Feifei, W. Ya-gang et al., "CJC-net: a cyclical training method with joint loss and Co-teaching strategy net for deep learning under noisy labels," Information Sciences, vol. 579, no. 11, pp. 186-198, 2021.

[20] Z. Mohammadnia and F. D. Moghadam, "Textbooks as resources for education for sustainable development: a content analysis," Journal of Teacher Education for Sustainability, vol. 21, no. 1, pp. 103-114, 2019.

[21] L. Wynter, A. Burgess, E. Kalman, E. H. Jack, and B. Jane, "Medical students: what educational resources are they using?” BMC Medical Education, vol. 19, no. 1, pp. 25-30, 2019.
[22] S. Ramoutar, "Open education resources: supporting diversity and sharing in education," TechTrends, vol. 65, no. 3, pp. 410-412, 2021.

[23] A. López-García and R. E. Mccleary, "Asymptotics of greedy energy sequences on the unit circle and the sphere," Journal of Mathematical Analysis and Applications, vol. 504, no. 1, Article ID 125269, 2021.

[24] Q. Liu, Y. Xue, and G. Chen, "Oscillation analysis based on trajectory modes decoupled in space and mode-energy-sequence Part One theoretical basis," Automation of Electric Power Systems, vol. 11, no. 1, pp. 1-10, 2019.

[25] N. Singh and P. M. Pradhan, "Achievable simultaneous time and frequency domain energy concentration for finite length sequences," IET Signal Processing, vol. 13, no. 8, pp. 736-746, 2019.

[26] C. Giardino, M. Unterkalmsteiner, N. Paternoster, G. Tony, and A. Pekka, "What do we know about software development in startups?" IEEE Software, vol. 31, no. 5, pp. 28-32, 2019.

[27] V. Hosseinpour, A. Saeidi, M.-J. Nollet, and M. Nastev, "Seismic loss estimation software: a comprehensive review of risk assessment steps, software development and limitations," Engineering Structures, vol. 232, no. 5, Article ID 111866, 2021.

[28] A. S. Bozkir and M. Aydos, "LogoSENSE: a companion HOG based logo detection scheme for phishing web page and E-mail brand recognition," Computers \& Security, vol. 95, no. 8, Article ID 101855, 2020.

[29] K. Lai, L. Wen, J. Lei, G. Chen, P. Xiao, and A. Maaref, "Codeword position index based sparse code multiple access system," IEEE Wireless Communications Letters, vol. 8, no. 3, pp. 737-740, 2019.

[30] T. Bluche, M. Primet, and T. Gisselbrecht, "Small-footprint open-vocabulary keyword spotting with quantized LSTM networks," no. 2, pp. 15-23, 2020, https://arxiv.org/abs/2002. 10851.

[31] M. Rivero-Contreras, P. E. Engelhardt, and D. Saldaa, "An experimental eye-tracking study of text adaptation for readers with dyslexia: effects of visual support and word frequency," Annals of Dyslexia, vol. 71, no. 6, pp. 1-18, 2021.

[32] A. Chaouch-Orozco, J. G. Alonso, and J. Rothman, "Individual differences in bilingual word recognition: the role of experiential factors and word frequency in cross-language lexical priming," Applied PsychoLinguistics, vol. 42, no. 2, pp. 1-28, 2020.

[33] Z. Fei, "Computer aided fencing sports wearable equipment based on FPGA microprocessor and sensors," Microprocessors and Microsystems, vol. 81, no. 8, Article ID 103693, 2020.

[34] T. . Murat, "Computer-controlled equipment for the direct measurement of the swell potential of expansive soils," Arabian Journal of Geosciences, vol. 12, no. 23, pp. 1-10, 2019.

[35] F. Ali and S. Khusro, "Content and link-structure perspective of ranking webpages: a review," Computer Science Review, vol. 40, no. 5, pp. 1-18, 2021.

[36] H. Wan, W. Ji, G. Wu et al., "A novel webpage layout aesthetic evaluation model for quantifying webpage layout design," Information Sciences, vol. 576, no. 5, pp. 589-608, 2021. 